\title{
Religious and Scientific importance of Vastu Shastra
}

\author{
Nimita Kanyal* \\ Sanskrit Department LSM Postgraduate College Pithoragarh, Uttarakhand, India \\ *Corresponding Author Email: nimitakanyal1982@gmail.com
}

Received: 1.12.2021; Revised: 19.12.2021; Accepted: 28.12.2021

(O) Society for Himalayan Action Research and Development

\begin{abstract}
:
The ancient sages created Vastu Shastra with great efforts for the protection of the human body and the biochemical reactions occurring in the body. In ancient times, the scientific architecture of the common man did not seem to be factual.Therefore, all this should be understood by them and if they feel right, they can accept it with devotion. From this point of view, Vastu Shastra was easily created by giving different shapes and names to these objects.In the present paper, scientific and religious analysis of Vastu Shastra has been presented in the context of Indian literature, Vedic and Valmiki Ramayana etc.
\end{abstract}

Keywords: Vastu Shastra, Religious, Scientific, Ramayan, Veda.

\section{वास्तुशास्त्र का धार्मिक तथा वैज्ञानिक महत्त्व}

\section{निमिता कन्याल}

\section{संस्कृत विभाग}

एल०एस०एम० रा०स्ना० महाविद्यालय पिथौरागढ ।

\section{सारांश}

मनुष्य के शरीर का और शरीर में घटने वाली जैव रासायनिक प्रक्रियाओं का बचाव हो, इसलिए प्राचीन ऋषि मुनियों ने बहुत कष्ट एवं परिश्रम के साथ वास्तुशास्त्र का निर्माण किया। प्राचीनकाल में जनसामान्य की वैज्ञानिक वास्तुदृष्टि तथ्यपरक नहीं लगती थी इसलिए यह सब उनकी समझ में आ जाये और उनको सम्यक् प्रतीत हो तथा उनकी ओर वे श्रद्धाभाव से देख सकें एवं उसे स्वीकार कर सकें। इस दृष्टि से इन वस्तुओं को विविध आकार तथा नाम देकर सहज रूप से वास्तुशास्त्र की रचना की गई। प्रस्तुत शोध पत्र में भारतीय साहित्य, वैदिक एवं वाल्मीकि रामायण आधरित सन्दर्भों के परिप्रेक्ष्य में वास्तुशास्त्र का धार्मिक एवं वैज्ञानिक तथ्यपरक विश्लेषण प्रस्तुत किया गया है।

शब्द कुंजी - वास्तुशास्त्र, धार्मिक, वैज्ञानिक, रामायण, वेद 
प्राचीनकाल में जनसामान्य की वैज्ञानिक वास्तुदृष्टि तथ्यपरक नहीं लगती थी इसलिए यह सब उनकी समझ में आ जाये और उनको सम्यक् प्रतीत हो तथा उनकी ओर वे श्रद्धाभाव से देख सकें एवं उसे स्वीकार कर सकें इस दृष्टि से इन वस्तुओं को विविध आकार तथा नाम देकर सहज रूप से वास्तुशास्त्र की रचना की गई।

"वास्तुमूर्तिः परमज्योतिः वास्तुदेवो परम्शिवः।

वास्तुदेवस्तु सर्वेषां वास्तुदेवं नमाम्यहम्।।"1

सृष्टि के पश्चात् जब मनुष्य का मानसिक विकास हुआ, और मनुष्य में ज्ञान और तर्क की बुद्धि का विकास हुआ तब वह अपने आस-पास की वास्तु का गम्भीर अध्ययन और अन्वेषण करने लगा। वह सोचने और तर्क करने लगा कि अग्नि और झंझावत (तूफानों) में किसका क्रोध निहित है? नदी का पानी कौन बहाता है ? पानी कौन बरसाता है? इत्यादि प्रश्नों के उत्तर में मनुष्य में ईश्वर के अस्तित्व की भावना जाग्रत हुई। तभी देवलोक की कल्पना का विकास हुआ और देवी शक्ति के प्रति मनुष्य के मन में भय का संचार हुआ। मनुष्य ने देवताओं को प्रसन्न करने के लिए उपासना और पूजा के विधान की कल्पना की। प्रत्येक मानव ने अपनी-अपनी कल्पना के अनुसार प्रकृति की शक्तियों के लिए भिन्न-भिन्न देवताओं और उनके रूपों की परिकल्पना की है। उसी के अनुरूप उसके धर्म का विकास हुआ। प्रत्येक धर्म के लोगों ने अपनी उपासना के रूप भी भिन्न-भिन्न निर्धारित किये। उसी के अनुसार साधारण निवास से भिन्न पवित्र स्थान बनाये और उसी के अनुकरण पर धार्मिक वास्तुओं का विकास हुआ।

भारत के निवासियों के धर्म का आदिस्वरूप क्या था? इस विषय में वैदिक वाङ्मय के अध्ययन से तात्कालिक धार्मिक व्यवस्था का विस्तृत ज्ञान प्राप्त होता है। वैदिक ऋचाओं में धार्मिक वास्तु के रूप में यज्ञवेदी और यज्ञशाला का उल्लेख मिलता है। उसे ही भारत का आदिवास्तु कहा जा सकता है। अनुमान किया जाता है कि तत्कालीन यज्ञवेदी मिट्टी और कुश के बने चबूतरे और शाला प्रारम्भिक कुटीर आदि रूप में रही होगी, तत्पश्चात् वेदिका को कलात्मक रूप दिया गया होगा। तैन्तरीय सहिता में पक्षी, रथ आदि के आकार की वेदिका बनाये जाने का निर्देश पाया जाता है। वाल्मीकि रामायण में भी एसे स्थलों का वर्णन प्राप्त होता है-

\section{"वेदिस्थलविधानानि चैत्यान्यायतनानि च।}

आश्रमस्यानुरूपाणि स्थापयामास राघवः।।"2

यज्ञशाला के वर्णन से ज्ञात होता है कि ये पवित्र धार्मिक भवन सम्भवतः बाँस और फूस के बनाये जाते थे। धार्मिक वस्तुओं का तात्पर्य मन्दिर आदि पूजा स्थलों से है। देवालय और मन्दिरों का निर्माण एक धार्मिक एवं पवित्र कार्य है। मन्दिर एक ऐसी संरचना है जो परिरूप के संतुलन और परस्पर सम्बन्धित विस्तार पर निर्भर करती है। मन्दिर के गर्भगृह में देवता की प्रतिष्ठा की जाती है और भक्तों के लिए देवता का ध्यान करते हुए परिक्रमा करने के लिए प्रदक्षिणा पथ होता है। गर्भगृह के ऊपर जो मीनार या बुर्ज होता है, उसे शिखर, गोपुरम् अथवा विमान कहते हैं। गर्भगृह एक आयताकार कक्ष में खुलता है, जिसे अन्तराल कहते हैं। मंडप में आने के लिए एक अर्धमंडप अथवा द्वार मंडप होता है। मंडप स्तम्भों पर खड़ा एक सभा भवन होता है। मन्दिर वास्तुशिल्प उसके अंगों के अनुसार तीन प्रकार का होता है, 
जिसे नागर, बेसर और दविड़ शैली कहते हैं। हिन्दू परम्परा के अनुसार मन्दिर एक मानव शरीर के अनुरूप होता है, जिसे परमात्मा का सचल रूप समझा जाता है। और उसके मध्य में जीव का वास होता है। मन्दिर का शीर्ष भाग सिर है। गर्भगृह उसकी ग्रीवा, सामने का मंडप उदर, परिक्रमा की दीवारें टांगें, और गोपुर चरण हैं। देवता की मूर्ति मन्दिर रूपी शरीर का जीव है। इस प्रकार मन्दिर के हर भाग की धारणा देवता के शरीर के रूप में की गयी है। रामायण में देव मन्दिरों से सम्बन्धित वर्णन प्राप्त होते हैं-देव मन्दिरों में चैत्य वृक्षों के नीचे या चौराओं पर जो पजूनीय देवताओं के मन्दिर हैं, उन्हें भक्ष्य भोज्य पदार्थ एवं दक्षिणा प्रस्तुत करनी चाहिए।

"देवायतन चैत्येषु सान्नभक्षयाः सदक्षिणाः।

उपस्थापयित्य्याः स्युर्माल्ययोग्याः पृथक्पृथक्।|'33

अपने आस-पास की सृष्टि एवं पर्यावरण में जो अनिष्ट शक्तियाँ काम करती रहती हैं, उनसे मनुष्य के शरीर का और शरीर में घटने वाली जैव रासायनिक प्रक्रियाओं का बचाव हो, इसलिए प्राचीन ऋषि मुनियों ने बहुत कष्ट एवं परिश्रम के साथ इस वास्तुशास्त्र का निर्माण किया। प्राचीनकाल में जनसामान्य की वैज्ञानिक वास्तुदृष्टि तथ्यपरक नहीं लगती थी। इसलिए यह सब उनकी समझ में आ जाये और उनको सम्यक् प्रतीत हो तथा उनकी ओर वे श्रद्धाभाव से देख सकें एवं उसे स्वीकार कर सकें। इस दृष्टि से इन वस्तुओं को विविध आकार तथा नाम देकर सहज रूप से वास्तुशास्त्र की रचना की गई। आज के आधुनिक काल की अपेक्षा प्राचीन काल में परमसत्ता में विश्वास रखने वाले, उसका अस्तित्व मानने वाले लोग निश्चित रूप से बहुत ज्यादा थे। इसी कारण हमारे ऋषि-मुनियों ने धार्मिकता तथा अध्यात्मिकता की नींव पर आधारित ग्रन्थों की रचना की है।

मनुष्य पृथ्वी का एक महत्तपूर्ण घटक है। प्रकृति की प्रत्येक घटना का,परिवर्तन का उसके जीवन पर उसके अनभिज्ञ होते हुए भी प्रभाव पडत़ा है। सूर्य मंडल का प्रमुख गृह सूर्य और उसके चारों ओर मंडलाकार भ्रमण करने वाले अन्य ग्रह और उसके उपग्रह इनसे प्राप्त होने वाली किरणों का मनुष्य शरीर की जैव रासायनिक क्रियाओं पर निश्चित रूप से प्रभाव पड़ता है। हमारे पर्यावरण में धनआयन तथा ऋणआयन दोनों समान बल के होते हैं। और उनका मनुष्य जीवन से बड़ा निकटतम सम्बन्ध रहता है। मानवीय मन में निर्माण होने वाले अच्छे विचार धनआयन हैं, तो बुरे विचार ऋणआयन होते हैं। मनुष्य हमेशा इनसे जुड़ा रहता है। हमारे श्वसन की हमेशा एक निश्चित गति होती है। किसी वास्तु में प्रवेश करने पर कभी-कभी इस गति में परिवर्तन आता है, इसका अनुभव कर लेना आसान है। यदि किसी नये स्थान पर प्रवेश करने से हमारी श्वास की गति बढ़ती है, तो उस जगह की निर्मिति में कोई दोष है, यदि धीरे-धीरे चलती है तो वास्तु शुभ है।

वास्तुविदों की मान्यता के अनुसार सूर्य, पृथ्वीवासियों के लिए ऊर्जा का एक महत्त्वपूर्ण स्रोत है। सूरज की किरणों में कुल मिलाकर सात रंग होते हैं। सूर्य के सप्तरंगीय किरणों का प्रभाव जमीन पर तथा वास्तुस्थान और सृष्टि पर अलग-अलग होता है। इन किरणों का प्रभाव हमारी भूमि पर पड़ना बहुत आवश्यक है। किसी वास्तु का निर्माण करते समय उसके ईशान कोण में भी काम करना योग्य नहीं है। 
इसलिए ईशान कोण में कूप नलिका, कुँआ, पानी का हौज हो तो शुभकारक है। इस हिस्से में शौचालय होता है तो ये किरणें दूषित हो जाती हैं।

वास्तुशास्त्र की दृष्टि से आग्नेय दिशा का अपना विशेष महत्त्व है। सूर्योदय के बाद अगर इन्फ़ारेड किरणों का पानी में अभिशोषण होता है तो मनुष्य जीवन के लिए बड़ा ही हानिकारक अथवा विपरीत परिणाम करने वाला होता है। इस विचार से वास्तुशास्त्र में अग्नि और जल को एक-दूसरे का विरोधी बताया गया है। सूर्योदय से लेकर सूर्यास्त तक किरणों का मनुष्य के शरीर में एवं निवास स्थान पर प्रभाव पड़ता है। पृथ्वी के गर्भ में एक महाचुम्बक रिथत है। मानवीय शरीर में भी चुम्बकीय परिणाम दिखाई देते हैं। हमारा सिर एक चुम्बक का उत्तरीय ध्रुव है। और हमारे पैर दक्षिण ध्रुव हैं। यदि हम दक्षिण की तरफ पैर फैलाकर सोते हैं तो उत्तर की तरफ हमारा सिर आता है। हमारे सिर का उत्तर ध्रुव और चुम्बकीय उत्तर ध्रुव एक दूसरे के पास आने से इनमें प्रत्याकर्षण होता है। इस प्रत्याकर्षण का हमारे रक्त सचं ललन पर विपरीत प्रभाव पडता है। जिस कारण हम प्रगाढ़ निद्रा का अनुभव नहीं कर पाते हैं। परन्तु इसके विपरीत हम दक्षिण दिशा की ओर मस्तक रखकर सोते हैं तो हमारे मस्तक में स्थित उत्तर ध्रुव पृथ्वी के चुम्बकीय दक्षिण ध्रुव के पास आता है और दो विरूद्ध ध्रुवों में आकर्षण होने के कारण मन शान्त रहकर गाढ़ निद्रा का अनुभव करते हैं। नैऋत्य दिशा में मध्यान के बाद जो किरणें पड़ती हैं, वे हानिकारक होती हैं। अतः यदि पश्चिम या दक्षिण दिशा में गड़े, पानी या उसकी व्यवस्था हो तो उनमें ये किरणें अभिशोषित होकर मनुष्य को बड़ी हानि या दुख पहुँचाती हैं। इससे बचाव के लिए दक्षिण और पश्चिम दिशा में कुछ ऊँची दीवारों या पेड़ आदि का होना शुभ माना जाता है। इनकी सहायता से हानिकारक किरणों से वास्तु का संरक्षण होता है। इसलिए पूर्व और उत्तर की दीवारों की अपेक्षा दक्षिण और पश्चिम की दीवार निश्चित रूप से ऊँची होनी चाहिए। दक्षिण और पश्चिम की ओर दरवाजे, खिड़कियाँ कम होनी चाहिए। जहाँ पर हम रहते हैं, वहाँ की निर्मिति वास्तुशास्त्र के अनुसार न होकर विपरीत हुई है तो हम वहाँ पर आवश्यक परिवर्तन द्वारा उन बाधाओं को दूर कर सकते हैं।

वाल्मीकि रामायण में विज्ञान का प्रमुख स्थान है। प्राचीन काल में खगोल अथवा नक्षत्र शासत्र का पर्याप्त अनुशीलन होता था। वाल्मीकि ने तत्सम्बन्धी प्रचुर सामग्री प्रस्तुत की है। दिन, रात, मास और वर्ष के चक्र की लोगों में बहुत पहले ही वैज्ञानिक जानकारी प्राप्त हो चुकी थी। कवि ने उसका स्थल-स्थल पर उल्लेख भी किया है। पुराकाल में लोग दिन, तिथि और नक्षत्र पंचांग के इन तीनों लक्षणों से भली-भाँति ज्ञात थे। राम का यौवराज्याभिषेक पुष्य नक्षत्र से युक्त चन्द्रमा के अधिपति वृहस्पति की प्रबलता के दिन प्रस्तावित हुआ था।

\section{वास्तुशास्त्र का मनोवैज्ञानिक पक्ष-}

"तन्मेमनः शिवसंकल्पमस्तु" की वैदिक अवधारणा से ओत-प्रोत वर्तमान में मनुष्य जाति ने आधुनिक एवं नवीन अन्वेषण किये। निर्माणकार्य के तन्त्र में मनुष्य ने यहाँ तक परिवर्तन कर लिया कि वास्तुशास्त्र सथापत्य शास्त्र का आधार स्तम्भ है, इस तथ्य को उसने विस्मृत कर दिया। कम से कम 
जगह में ज्यादा सुविधायें कैसे लाई जायें, इसके बारे में वह सोचता चला गया। परन्तु इन बातों का वास्तु या आस-पास की वस्तुओं पर क्या प्रभाव पडत़ा है, इस ओर उसने ध्यान नहीं दिया। भवन सुशोभित करने के लिए मानव ने गोल, त्रिकोण, चतुष्कोण, त्रियककोण (तिरछ) एवं ऊँचे प्रकार के भवनों का निर्माण किया। इससे सिर्फ भवन आकार और सौन्दर्य इन सब की तरफ ही उसका ध्यान गया। इनके लिए बहुमूल्य निर्माण/साहित्य का भी निर्माण किया गया। परन्तु उन भवनों को बनवाते समय वास्तुशास्त्र के नियमों का पालन न होने के कारण इस प्रकार से निर्मित भवनों में अपयश, दुःख, संघर्ष आदि का सामना मनुष्य को करना पड़ता है। दिनभर की थकान से भरपूर और यातायात पूर्ण जीवन के बाद यदि किसी व्यक्ति को अपने घर में मनः शांति नहीं मिल रही है, तो जीवन का क्या अर्थ ? कोई भी घर केवल पत्थर, ईंट, सीमेन्ट या चूने से तैयार नहीं होता, बल्कि उसमें घर के लोगों का मानसिक साहचर्य, मानसिक एकात्मकता का भाव भी उस स्थान को घर का स्वरूप प्रदान करता है। निवास स्थान बनवाने के लिए बहुत सारा धन व्यय होने के बावजूद यदि वहाँ पर मानसिक सन्तोष की प्राप्ति नहीं हो रही, या कहीं पर किसी भी विषय में प्रगति नहीं हो रही हो, तो वह वास्तु अपूर्ण है। प्राचीनकालखण्ड में वास्तुशास्त्र के नियमों के अनुसार ही गृह निर्माण कार्य होता था। हमारे श्रेष्ठ ऋषि मुनियों ने वास्तुशास्त्र का मेल एवं मानव का साहचर्य पर्यावरण के साथ दर्शाया था। यह मेल दो विश्वयुद्धों और उसके बाद चली आधुनिकीकरण की लहरों में पूरी तरह नष्ट हो गया। प्राचीनकाल में हमारा देश सोने की चिड़िया कहलाता था, परन्तु आज हमारा देश सामाजिक, आर्थिक, राजनीतिक तथा मानसिक विपत्तियों से घिरा हुआ है।

आज यदि कोई भी व्यक्ति नया घर बनाते समय वास्तुशास्त्र के नियमों का अधिकाधिक आश्रय लेगा और उसके अनुसार ही निर्माण कार्य करेगा तो उसे मनः शान्ति का लाभ निश्चित रूप से होगा, उसके साथ-साथ उसकी समृद्धि में भी वृद्धि होगी। एसे भी सन्दर्भ मिलते हैं कि-कई निवास स्थानों में कुछ लोगों की कई पीढ़ियों ने अपना जीवन सुख और समृद्धिपूर्वक व्यतीत किया। इन वास्तुओं का परिशीलन करने पर ज्ञात होता है कि इन भवनों का निर्माण वास्तुशास्त्र के नियमों का पालन करते हुए किया गया है। प्रकृति के पंच महाभूतों को भी अपने निवास स्थान में उचित, यथायोग्य स्थान देकर प्राकृतिक वातावरण की अनन्त, अदृश्य शक्तियों के साथ सामंजस्य रखना मानव के लिए आवश्यक है, जिसकी जानकारी वास्तुशास्त्र के माध्यम से मानव को होती है, ऋग्वदे की एक ऋचा में वास्तुशास्त्रीय दृष्टि से निर्मित गृह द्वार को रोग रहित, सर्वसुख तथा मंगलप्रद कहा गया है।

"वास्तोष्पते प्रति जानीह्यस्मान् स्वावेशो अनमीवो भवा नः।

यत् त्वमे हे प्रति तन्नो जुषस्व शन्नो भव द्विपदे शं चतुष्पदे।।"4

\section{संदर्भ सूची}

1- वास्तुशास्त्रानुसार भवन निर्माण- डॉ० मुरलीधर राव पृ०24

2- वाल्मिकी रामायण अयोध्याकाण्ड- $56 / 33$

$3-$ वाल्मिकी रामायण अयोध्याकाण्ड- $3 / 18$

$4-$ ॠग्देव $-7 / 54 / 1$ 